individual consumption below cerrtain levels, there would seem a good case for making the manufacturers disclose the nature and quantity of artificial sweeteners added to soft drinks. This can operate only in their own interests, and, while most consumers will pay no heed to the information, those who are concerned about their own or their children's health will at least be able to make the calculations necessary to assure themselves that their consumption is within recommended limits.

It is satisfactory to know that the British Industrial Biological Research Association is keeping a watchful eye on cyclamates as on other actual and potential food additives. Not only does the association publish regular critical and unprejudiced surveys of published work from all parts of the world but it is engaged in some of the crucial animal tests for safety. The Government has shown its confidence in the value of this research association in protecting public health by the size of the grant which it awards.

\section{Attack on Cholera}

Up to the middle of this century cholera was being gradually contained within its endemic areas in India and East Pakistan, but large epidemics in Egypt in 1947 and in Thailand in 1958 showed that the smouldering fires could still light up conflagrations in other countries. These epidemics attracted attention from groups of American workers, whose studies shed new light on the pathogenesis and treatment of cholera. When, in more recent years, cholera caused by the El Tor variant ${ }^{1}$ of the vibrio began to spread through the Far East from an endemic focus in Indonesia and then swept back westwards to involve India (where it has supplanted infection with the classical strain), the Middle East, and southern Russia, world-wide interest was aroused, and now large-scale studies into the pathogenesis, epidemiology, and control of the disease are being sponsored by the World Health Organization, the National Institutes of Health in the U.S.A., and similar bodies in India, Japan, and elsewhere.

The Cholera Research Laboratory established under the joint auspices of the South-east Asia Treaty Organization (S.E.A.T.O.) and Pakistan, with strong American support, has been in the forefront of investigations into all aspects of cholera and has lately published a series of three papers on controlled vaccine trials carried out in 1966-7.23 Having shown in earlier studies that a vaccine of high antigenic potency could give good protection (60-80\% effectiveness), and that cholera occurs predominantly in children, with the highest case rate among those under 5, the team at the Cholera Research Laboratory designed a new vaccine trial in a more extended population but limited to children aged 3 months to 14 years. A commercial cholera vaccine with a combined diphtheria and tetanus toxoid as a control was given in one- and two-dose schedules to some 40,000

British Medical fournal, 1966, 1, 1555.

Mosley, W. H., et al., Bulletin of the World Health Organization, $1969,40,177$.

s Mosley, W. H., McCormack, W. M., Ahmed, A., Alauddin Chowdhury, A. K. M., and Bauri, R., Bulletin of the World Health Organization, 1969, 40, 187.

- McCormack, W. M., Mizanur, A. S. M., Alauddin Chowdhury, A. K. M., Mosley, W. H., and Phillips, R. A., Bulletin of the World Health Organization, $1969,40,199$.

- World Health Organization Technical Reports Service, 1969, No. 414.

- Freter, R., in Proceedings of the Cholera Research Symposium, ed. O. A. Bushnell, p. 222. Washington, Public Health Service Publication No. 1328. 1965. children in an endemic rural area. The children were stratified in three age groups, 0-4 years, 5-9 years, and 10-14 years, and were assigned in strict alternation to one of three schedules: two doses of diphtheria and tetanus toxoid, one dose of cholera vaccine with one dose of diphtheria and tetanus toxoid, and two doses of cholera vaccine. Two children were assigned to the double dose of cholera vaccine for one each on the other two schedules. All vaccines were given by a jet-injector, and the vaccination programme was carried out during 20 September to 17 November 1966.

The first cholera cases (acute diarrhoea detected by daily family visits and confirmed bacteriologically) occurred in November, and the epidemic wave quickly reached its peak in December-January, gradually subsiding over the next four months. From analyses of the findings over the sevenmonth period it was apparent that two doses of the cholera vaccine were needed to give maximum protection (91\%) to the youngest age group, that this protection lasted for only three months, and that one dose gave at least as good protection as two doses to children aged 5-14 years, with immunity waning in the later months of the epidemic. The data indicate that among preschool children highly exposed to infection in an endemic area two doses of a cholera vaccine will give only short-lived protection, whereas in older children one dose will act as a not very efficient booster in an age group already well "salted" by latent infection. Indeed, it is reckoned from this and other studies that the ratio of clinical to latent cholera infection in an endemic area may range from $1: 20$ to $1: 100$.

As part of this vaccine study specimens of blood collected by finger-tip puncture in a capillary pipette were taken from random samples of the test community before vaccination and during and after the epidemic season. The sera were tested for vibriocidal antibody by a technique which seemed to give reproducible results. Earlier it had been shown that there was a' steady rise in vibriocidal antibody titres with increasing age and that this rise in antibody was correlated inversely with a fall in the case-rate. In the current study the antibody titres in the age group 0-4 years were significantly higher in the children given two doses than in those given one dose, but there was no such difference in the older age groups. These findings, with other evidence, indicate that the level of vibriocidal antibody in a population is a good measure of the degree of immunity to cholera whether naturally or artificially acquired. In the third paper in this series $^{4}$ the authors assessed the clinical severity of cholera in relation to the vaccination status and found no correspondence between these two measurements. This suggests that good antibody titres will not protect individual children against the onslaught of a really heavy infection. Thus in its epidemiology cholera has many features in common with other acute infections.

There have so far been reports on six controlled trials Iwith 13 different cholera vaccines in three areas (East Pakistan, Calcutta, and the Philippines), and the summation of the results shows that some cholera vaccines give protection in the range of $40-80 \%$ for a period of three to six months. This level of achievement is not satisfactory as a public health measure for the control of the disease, particularly in non-endemic areas. In endemic areas, one booster dose for schoolchildren given immediately before the expected epidemic season could be recommended if the machinery for large-scale immunization is available. But clearly improvements will have to be made in the antigenicity of parenteral vaccine-for example, by using purified frac- 
tions with an adjuvant, or by a combination of whole-cell vaccine and toxoid. ${ }^{5}$ Alternatively the use of live attenuated vaccines, given by mouth as recommended by R. Freter, ${ }^{6}$ will have to be explored. Meanwhile the risk to countries with poor sanitation from the chance importation of cholera by persons with mild or inapparent infection will need to be met by constant vigilance.

\section{Tropical Splenomegaly Syndrome}

In malarious zones, of the tropics a familiar syndrome is massive splenomegaly in adults for which no underlying cause is apparent. The condition is not due to active malarial infection, since parasitaemia is scanty or absent and malaria pigment is not found in biopsy material from liver or spleen. Nevertheless, malaria probably plays some part in the aetiology of the disease. ${ }^{12}$

The disease occurs only in malarious areas and has become less frequent where malaria control is effective. Serum samples show high titres of malarial antibody detected by the fluorescent technique, ${ }^{3}$ and in a clinical trial in Nigeria continuous antimalarial chemotherapy was associated with a regression of splenomegaly. ${ }^{4}$ In addition, recent studies in Uganda indicate that the syndrome may not occur in people heterozygous for sickle-cell haemoglobin, which confers protection from severe Plasmodium falciparum malaria. ${ }^{5}$ A reasonable hypothesis is that the tropical splenomegaly syndrome represents an abnormal immunological reaction to malarial antigen, owing to genetic or acquired host factors or to inoculation with an unusual species of malaria parasite, a combination of species, or an unusual frequency of infection. In support of this idea are the common findings of lymphocytic proliferation in the spleen and in the sinusoids and portal tracts of the liver and occasionally in the bone marrow, together with hyperplasia of reticuloendothelial cells and a high concentration of the serum immunoglobulin, $\operatorname{IgM}^{6}$

Working in Uganda, Dr. J. L. Ziegler, Dr. M. H. Cohen, and Professor M. S. R. Hutt have investigated immunological reactions in patients with this disorder, and report their findings at page 15 of the B.M.F. this week. Patients with the tropical splenomegaly syndrome react normally to a variety of skin-test antigens, and their lymphocytes transform normally in culture in response to phytohaemagglutinin. Furthermore, they make antibodies normally in response to a selected foreign antigen. The results show that the patients do not suffer from immunological deficiency as judged by the usual tests-though they do not indicate whether these people would have normal immunological responses to malarial antigens.

J. V. Dacie and his colleagues ${ }^{7}$ have recently pointed out that a syndrome of massive splenomegaly affecting people in Britain who have not been exposed to malaria has many

\footnotetext{
1 British Medical fournal, 1967, 4, 614.

2 Pitney, W. R., Transactions of the Royal Society of Tropical Medicine and Hygiene, 1968, 62, 717.

3 Gebbie, D. A. M., et al., Lancet, 1964, 2, 392

- Watson-Williams, E. J., and Allan, N. C., British Medical fournal, $1968,4,793$.

5 Hamilton, P. J. S., et al., Lancet, 1969, 2, 109.

6 Wells, J. V., Clinical and Experimental Immunology, 1968, 3, 943.

Dacie, J. V., Brain, M. C., Harrison, C. V., Lewis, S. M., and

Worlledge, S. M., British fournal of Haematology, 1969, 17, 317.

8 Pryor, D. S., Quarterly fournal of Medicine, 1967, 36, 321.

Richmond, J., Donaldson, G. W. K., Williams, R., Hamilton, P. J. S.

10 Pryor, D. S., Quarterly fournal of Medicine, 1967, 36, 337.
}

of the clinical and histological features of the tropical splenomegaly syndrome. They go on to suggest that in both conditions the cellular proliferation in spleen and liver is a response to antigenic stimuli, in the non-tropical cases possibly to autoantigens and in the tropics to repeated infection with malarial or other antigens. This unifying concept suggests that the abnormality is in the response of the host and that no one particular antigen is involved. And it would explain why, usually, only a small proportion of people develop the tropical splenomegaly syndrome when the entire population is exposed to the same degree of malaria inoculation. In certain inbred populations, such as inhabit the Upper Watut valley in New Guinea, where there is a high incidence of tropical splenomegaly syndrome ${ }^{8}$ it could be postulated that this host factor is genetically acquired.

The tropical splenomegaly syndrome causes illness and death. Anaemia is often severe, and the lowest haemoglobin values are found in the patients with the largest spleens. The anaemia is due to a combination of increased red-cell destruction in the spleen, splenic pooling of red cells, and an expanded plasma volume. ${ }^{910}$ Splenectomy restores the life span of the red cells to normal, removes the splenic pool, and is associated with a reduction in the plasma volume. Nevertheless, this operation should not be undertaken in tropical countries without considering the increased risk to the patient of fulminating malaria or bacterial infections, for he may live far from medical supervision. Continuous treatment with proguanil ${ }^{4}$ has proved effective in one clinical trial in reducing the size of the spleen and improving haemoglobin values. Trials with proguanil or alternative forms of antimalarial chemotherapy are indicated in other areas, since there is some evidence that the tropical splenomegaly syndrome may not everywhere be the same condition. Chemotherapy, if shown to be generally effective, would appear to be preferable to splenectomy for those patients whose clinical condition warrants treatment and for whom continuing medical care is possible.

\section{The Team}

Teamwork between doctors, nurses, and other staff has for long been an accepted part of the hospital scene. However, the great majority of health care is practised in a domiciliary setting, and here the evolution of a team approach is a comparatively recent development. ${ }^{1}$ Similarly, though there are notable exceptions to the generalization, there has been all too little coming and going between the various members of the team who provide care in hospitals and their colleagues in the communities which these hospitals serve.

The slow development of the team approach in the community stems partly from defects in medical education, partly from the administrative separateness of the general practitioner and public health services, but mainly from attitudes which had their origins in the days when these two branches of the medical profession were competitors in the provision of care for certain groups. Such entrenched attitudes die hard, but one of the best ways of altering them is by discussion between those directly concerned. An opportunity for this arose last month at a conference in Edinburgh on "The Team," sponsored by four nursing organizations and by the Scottish branches of the Royal College of General Practitioners and of the Society of Medical Officers of Health. 